Original Paper http://ajol.info/index.php/ijbes http://indexmedicus.afro.who.int

\title{
Performances zootechniques du poulet de chair soumis à un régime à base de résidus de semoule de manioc supplémenté par la spiruline (Spirulina platensis)
}

\author{
Jean Raphaël KANA ${ }^{1 *}$, Ahmat Djefil HASSAN ${ }^{1}$, Tadjong Ruben NGOUANA ${ }^{1}$, \\ Kuietche Hervé MUBE ${ }^{1}$, Alexis TEGUIA ${ }^{1}$, Yves TEFACK ${ }^{2}$ et Henri René ZAMBOU ${ }^{3}$ \\ ${ }^{I}$ Département des Production Animales, Faculté d'Agronomie et des Sciences Agricoles, \\ Université de Dschang, BP: 70 Dschang, Cameroun. \\ ${ }^{2}$ Centre d'Incubation Pilote de la CCIN (Chambre de Commerce, d'Industrie, des Mines et de l'Artisanat), \\ Douala, Cameroun. \\ ${ }^{3}$ EQUAVET Group SARL, Douala, Cameroun. \\ *Auteur correspondant, E-mail : kanajean@yahoo.fr
}

\section{RESUME}

Cette étude a été réalisée pour évaluer les performances zootechniques d'un régime alimentaire à base de résidus de semoule de manioc supplémentés par la spiruline chez les poulets de chair. Trois (03) groupes de poussins de souche Arbor Acres soumis aux rations témoins supplémentées ou non par la spiruline ont été comparés à neuf (09) autres groupes soumis aux rations dans lesquelles le maïs a été substitué par les résidus de semoule de manioc aux taux de 50,75, et $100 \%$ et supplémentés par 0,5 ou $1 \%$ de spiruline. Les principaux résultats ont montré que tous les paramètres de croissance se détériorent avec le taux croissant d'incorporation de résidus de semoule de manioc supplémentés ou non par la spiruline. L'indice de consommation a augmenté de l'ordre de 10; 10 et $15 \%$ respectivement avec 50, 75 et 100\% de substitution du maïs dans la ration. Le rendement carcasse, le poids relatif de la tête, des pattes, du foie, du cœur, du pancréas et de la graisse abdominale évolue en dents de scie d'un traitement à l'autre. Les résidus de semoule de manioc peuvent avantageusement être supplémentés par la spiruline au taux de 0,5\% dans l'aliment des poulets de chair. (c) 2015 International Formulae Group. All rights reserved.

Mots clés: Maïs, manioc, poulets de chair, spiruline.

\section{Zootechnical performances of broiler chickens fed on Spirulina platensis-supplemented cassava root waste based diet}

\begin{abstract}
This study investigated the growth performances of broiler chickens fed on spirulina-supplemented cassava root waste based diet. Three (03) groups of Abor Acres chicks fed on control diets supplemented or not with spirulina were compared to nine (09) other groups fed on diets in which 50, 75 and 100\% of maize were replaced by cassava root waste supplemented or not with 0.5 and $1 \%$ spirulina in a completely randomized design. Results revealed that growth parameters including feed intake, weight gain and feed conversion ratio
\end{abstract}


(FCR) depressed with increasing level of cassava root waste in the diet. FCR was poorer by 10,10 and $15 \%$ respectively with 50,75 and $100 \%$ of maize replaced with cassava root waste. Nevertheless, supplementing with spirulina, irrespective to the level of cassava root waste inclusion, demonstrated a production advantage. Carcass yield and relative weight of organs varied from one experimental diet to another. In conclusion, cassava root waste can be beneficially supplemented with spirulina at $0.5 \%$ in broiler diets.

(C) 2015 International Formulae Group. All rights reserved.

Keywords: Broiler chickens, cassava, maize, spirulina.

\section{INTRODUCTION}

L'homme et les industries agroalimentaires sont en perpétuelle concurrence avec les animaux d'élevage pour le maïs produit en quantités très limitées en Afrique. L'une des solutions pour les fermiers serait d'utiliser les ressources alimentaires alternatives localement disponibles qui n'entrent pas directement dans l'alimentation humaine. Parmi ces ressources figurent en bonne place les sous-produits tels que les épluchures et les résidus de semoule de manioc. Jusqu'à présent, l'incorporation de ces sous-produits dans l'aliment du poulet comme source d'énergie a toujours donné des résultats inférieurs à ceux du maïs (AwahNdukum et al., 2008; Kana et al., 2014). Les travaux de Kana et al. (2014) portant sur la substitution du maïs par les résidus de semoule de manioc supplémentés par l'huile de palme dans l'aliment du poulet de chair a permis d'obtenir des résultats supérieurs à ceux produits avec l'aliment sans huile mais encore faible par rapport à ceux produits avec le maïs. Parmi les raisons évoquées, il y a la pauvreté de ce sous-produit en nutriments essentiels tels que les protéines et sa déficience en tous les autres nutriments. Il est nécessaire de supplémenter les rations formulées à base du manioc et de ses sousproduits avec les protéines de haute valeur biologique, les minéraux et les vitamines à des taux plus élevés que lorsqu'on formule avec le maïs uniquement. La spiruline, en raison de sa forte concentration en nutriments de haute valeur, se présente comme un supplément idéal pour ce genre de sous-produits.

La spiruline est une algue bleu-vert qui doit son nom à sa forme spirale. Elle se développe dans les eaux saumâtres, salées, riches en bicarbonate de sodium communément appelé natron. Elle est l'aliment le plus riche actuellement connu en protéines (60 à 70\%). Elle est deux fois plus riche que le soja et trois fois plus que la viande maigre. Sa protéine est d'excellente qualité puisqu'elle contient tous les acides aminés essentiels et elles sont facilement assimilables par l'organisme (Pierlovisi, 2008). L'organisme les assimile quatre fois plus vite et mieux que les protéines de la viande et du fromage (Jourdan, 2011). Très riche en vitamines (A, B1, B2, B12, E) et en fer assimilable, elle contient aussi du calcium, du phosphore, du magnésium en quantité comparable aux céréales et au lait de vache (Jourdan, 2011). Selon Razafindrajaona et al. (2008), l'incorporation de la spiruline au taux de $100 \mathrm{mg} / \mathrm{kg}$ dans l'aliment améliore sa qualité nutritionnelle et les performances de croissance des poulets entre 11 et $15 \%$ par rapport à l'aliment témoin sans spiruline. En considérant ces vertus, son utilisation comme source de protéines pourrait permettre de mieux valoriser l'aliment à base des sousproduits pauvres en nutriments comme les résidus de semoule de manioc. Cette étude a pour objectif de contribuer à la valorisation des sous-produits de manioc comme source d'énergie alimentaire chez le poulet de chair dans le but de réduire durablement la compétition entre l'homme, l'animal et les industries pour les céréales.

\section{MATERIEL ET METHODES \\ Site de l'étude}

Cette étude a été menée à la Ferme d'Application et de Recherche (FAR) de l'Université de Dschang, Cameroun entre les mois de juin et juillet 2014. Cette ferme est 
située à $05^{\circ} 26^{\prime}$ de latitude Nord, $10^{\circ} 26^{\prime}$ de longitude Est, et culmine à une altitude moyenne de $1420 \mathrm{~m}$. Dschang est localisée dans la zone agro-écologique des hautes terres de l'Ouest du Cameroun. Le climat qui y règne est équatorial de type camerounien d'altitude et se caractérise par deux saisons, une saison de pluie qui va de mi-mars à minovembre et une saison sèche qui va de minovembre à mi-mars. La pluviométrie moyenne annuelle est de $2000 \mathrm{~mm}$ et la température moyenne se situe autour de 21 ${ }^{\circ} \mathrm{C}$. L'insolation moyenne annuelle est de 1873 heures et l'humidité relative moyenne est de 76,8\% (Kana et al., 2014).

\section{Matériel animal}

Dans cette étude, 504 poussins d'un jour, de souche Abor Acres, de poids moyen $42 \mathrm{~g}$, ont été repartis au hasard dans 36 unités expérimentales de 14 poussins chacune ( 7 mâles et 7 femelles) et élevés sur litière faite de copeaux de bois blanc. Les poussins ont été vaccinés contre la bronchite infectieuse $(\mathrm{H} 120)^{\circledR}$ et la maladie de Newcastle (Hitchner $\mathrm{B} 1)^{\circledR}$ le $7^{\text {eme }}$ jour avec un rappel le $18^{\text {ème }}$ jour et contre la maladie de Gumboro (IBA Gumboro) $^{\circledR}$ le $10^{\text {ème }}$ jour. Un antistress (Aliseryl ws) ${ }^{\circledR}$ a été servi dans l'eau de boisson pendant les 3 premiers jours dès l'entrée des poussins à la poussinière. Un anticoccidien $(\text { Vetacox })^{\circledR}$ et des vitamines (Amintotal) $^{\circledR}$ ont été servis dans l'eau de boisson 3 jours de suite chaque semaine. L'antistress a été administré après le transfert des poussins de la poussinière au bâtiment finition à l'âge de 21 jours.

\section{Rations alimentaires et dispositif expérimental}

Trois rations témoins $\left(\mathrm{R} 0, \mathrm{R} 0_{(0,5)}\right.$ et $\left.\mathrm{R} 0_{(1)}\right)$ avec ou sans spiruline ont été fabriquées avec du maïs comme seule source d'énergie. Neuf autres rations ont été respectivement fabriquées en substituant le maïs par des résidus de manioc aux taux de 50, 75, et $100 \%$ supplémentées ou non (R0, R75 et R100) par $0,5 \%\left(\mathrm{R} 0_{(0,5)}, \quad \mathrm{R} 75_{(0,5)}\right.$ et $\left.\mathrm{R} 100_{(0,5)}\right)$ et $1 \%$
$\left(\mathrm{R} 0_{(1)}, \quad \mathrm{R} 75_{(1)}\right.$ et $\left.\mathrm{R} 100_{(1)}\right)$ de spiruline au démarrage (Tableau 1) et en finition (Tableau 2). Chacune des 12 rations expérimentales a été affectée au hasard à 3 unités expérimentales dans un dispositif complètement randomisé avec 3 répétitions. La composition des résidus de manioc est la suivante: $0,34 \%$ de matière azotée totale, $0,39 \%$ de matière grasse, $1,89 \%$ de cellulose brute et $3754,79 \mathrm{kcal} / \mathrm{kg}$ d'énergie métabolisable. L'aliment et l'eau étaient servis ad libitum pendant toute la période de l'essai. Les oiseaux ont été élevés sur litière à une densité de 20 poussins $/ \mathrm{m}^{2}$ jusqu'à l'âge de 21 jours puis à la densité de 10 poulets $/ \mathrm{m}^{2}$ jusqu'à l'âge de 49 jours.

\section{Collecte des données}

Au début de l'essai et tous les 7 jours par la suite, les oiseaux de chaque unité expérimentale ont été pesés individuellement à jeun. Le gain de poids hebdomadaire a été obtenu en faisant la différence entre 2 poids hebdomadaires moyens consécutifs. A l'âge de 49 jours, 8 poulets par traitement (4 mâles et 4 femelles) ont été sélectionnés au hasard et soumis à une diète de 24 heures, puis pesés, saignés, plumés et éviscérés tel que préconisé par Jourdain (1980). Le poids relatif de chaque organe (gésier, foie, cœur) par rapport au poids vif a été calculé. La longueur de l'intestin a été mesurée de la loupe duodénale au cloaque à l'aide d'un mètre ruban et la densité de l'intestin (poids de l'intestin/ longueur de l'intestin) a été calculée.

\section{Analyses statistiques}

Les données sur la consommation alimentaire, le poids vif, l'indice de consommation et les caractéristiques de la carcasse ont été soumis à l'analyse de la variance (ANOVA) suivant un dispositif complètement randomisé. Le logiciel SPSS 12.0 (Statistical Package of Social Sciences) a été utilisé pour les analyses. Lorsqu'il existait des différences entre les moyennes, le test de Duncan a été appliqué pour les séparer au seuil de signification de $5 \%$. 


\section{RESULTATS}

Le Tableau 3 résume les effets de la spiruline et du taux d'incorporation des résidus de semoule de manioc en remplacement du maïs dans l'aliment sur la consommation alimentaire, le poids vif, le gain de poids et l'indice de consommation cumulé des poulets de chair. Au-delà de $50 \%$ de résidus de semoule dans l'aliment, la consommation alimentaire a baissé avec l'augmentation du taux d'incorporation de spiruline. A l'exception de l'indice de consommation, tous les autres paramètres ont baissé avec le taux d'inclusion croissant des résidus de semoule supplémentés ou non par la spiruline dans la ration pendant la phase finition et sur toute la période de l'étude. Par contre, à la phase démarrage, la ration témoin supplémentée par $1 \%$ de spiruline a induit une augmentation significative du poids vif et du gain de poids des poussins comparée à toutes les autres rations quel que soit le taux d'incorporation des résidus de semoule. A taux d'incorporation de résidus de semoule égal, les rations supplémentées par 0,5 et $1 \%$ de spiruline ont permis d'enregistrer les poids vifs et les gains de poids plus élevés $(\mathrm{P}<0,05)$ comparées aux rations sans spiruline. Par rapport à la ration témoin $\mathrm{R} 0$ sans résidus de semoule, l'indice de consommation a augmenté de l'ordre de 10; 10 et $15 \%$ respectivement avec 50; 75 et $100 \%$ de substitution du maïs dans la ration. Toutefois, cette augmentation a été seulement de l'ordre de $6 ; 1,8$ et $7 \%$ lorsque les résidus de semoule ont été supplémentés par $0,5 \%$ de spiruline (Figure 1).

Le Tableau 4 présente l'effet du taux d'incorporation des résidus de semoule de manioc dans l'aliment sur les caractéristiques de la carcasse. Il apparait lorsqu'on considère le même taux de résidus de semoule que, le rendement carcasse, le poids relatif de la tête, des pattes, du foie, du cœur, du pancréas et de la graisse abdominale évoluent en dents de scie d'un traitement à l'autre. A taux de spiruline égal et à l'exception du poids relatif du cœur et du pancréas, tous les traitements sont comparables pour le rendement carcasse et le poids des organes quel que soit le taux de résidus de semoule dans la ration.

Tableau 1: Composition des rations expérimentales en phase démarrage.

\begin{tabular}{|c|c|c|c|c|}
\hline Ingrédients (\%) & R0 & R50 & R75 & R100 \\
\hline Maïs & 54,0 & 27,0 & 13,5 & 0,0 \\
\hline Remoulage & 3,0 & 0,5 & 1,5 & 0,0 \\
\hline Résidus de semoule & 0,0 & 27,0 & 40,5 & 54,0 \\
\hline Huile de palme & 3,0 & 3,0 & 3,0 & 3,0 \\
\hline Tourteau de coton & 7,0 & 8,5 & 7,5 & 8,0 \\
\hline Tourteau de soja 49 & 23,0 & 23,0 & 21,0 & 22,0 \\
\hline Farine de poisson 60 & 4,0 & 5,0 & 4,0 & 4,0 \\
\hline Farine de sang & 0,0 & 0,0 & 3,0 & 3,0 \\
\hline Coquillage & 1,0 & 1,0 & 1,0 & 1,0 \\
\hline CMAV 5\%* & 5,0 & 5,0 & 5,0 & 5,0 \\
\hline Total & 100,0 & 100,0 & 100,0 & 100,0 \\
\hline \multicolumn{5}{|c|}{ Caractéristiques chimiques calculées } \\
\hline Energie métabolisable ( $\mathrm{kcal} / \mathrm{kg})$ & 3056,16 & 2951,28 & 2945,57 & 2890,8 \\
\hline Protéines Brutes (\%) & 23,43 & 22,66 & 22,56 & 22,21 \\
\hline Energie/Protéine & 130,44 & 130,26 & 130,51 & 130,11 \\
\hline Lysine (\%) & 1,4 & 1,4 & 1,48 & 1,4 \\
\hline Méthionine (\%) & 0,48 & 0,6 & 0,44 & 0,43 \\
\hline Calcium (\%) & 1,1 & 1,23 & 1,18 & 1,21 \\
\hline Phosphore disponible (\%) & 0,46 & 0,48 & 0,45 & 0,44 \\
\hline
\end{tabular}


Tableau 2: Composition des rations expérimentales en phase finition.

\begin{tabular}{|c|c|c|c|c|}
\hline Ingrédients (\%) & R0 & R50 & R75 & R100 \\
\hline Maïs & 60,0 & 30,0 & 15,0 & 0,0 \\
\hline Remoulage & 2,0 & 1,7 & 3,0 & 2,3 \\
\hline Résidus de semoule & 0,0 & 30,0 & 45,0 & 60,0 \\
\hline Huile de palme & 3,0 & 3,0 & 3,0 & 3,0 \\
\hline Tourteau de coton & 8,5 & 5,5 & 0,0 & 0,0 \\
\hline Tourteau de soja 49 & 13,0 & 15,5 & 17,0 & 17,0 \\
\hline Farine de poisson 60 & 2,5 & 3,0 & 6,0 & 5,7 \\
\hline Farine de sang & 3,0 & 4,5 & 5,0 & 6,0 \\
\hline Coquillage & 1,0 & 1,0 & 1,0 & 1,0 \\
\hline Farine d'os & 2,0 & 0,8 & 0,0 & 0,0 \\
\hline CMAV 5\%* & 5,0 & 5,0 & 5,0 & 5,0 \\
\hline Total & 100,0 & 100,0 & 100,0 & 100,0 \\
\hline \multicolumn{5}{|c|}{ Caractéristiques chimiques calculées } \\
\hline Energie métabolisable $(\mathrm{kcal} / \mathrm{kg})$ & 3076,95 & 3024,95 & 3027,75 & 2984,26 \\
\hline Protéines Brutes $(\%)$ & 21,23 & 20,95 & 20,95 & 20,61 \\
\hline Energie/Protéine & 144,96 & 144,36 & 144,55 & 144,81 \\
\hline Lysine (\%) & 1,2 & 1,30 & 1,5 & 1,5 \\
\hline Méthionine (\%) & 0,42 & 0,42 & 0,44 & 0,42 \\
\hline Calcium $(\%)$ & 1,5 & 1,3 & 1,3 & 1,3 \\
\hline Phosphore disponible $(\%)$ & 0,64 & 0,52 & 0,50 & 0,49 \\
\hline
\end{tabular}

"CMAV 5\%: Complexe Minéral Azoté et Vitaminique , Protéine brute $=40 \%$, Lysine $=3,3 \%$, Méthionine $=2,40 \%$, Calcium $=8 \%$, Phosphore $=2,05 \%$, Energie métabolisable $=2078 \mathrm{kcal} / \mathrm{kg}$. 
J. R. KANA et al. / Int. J. Biol. Chem. Sci. 9(5): 2607-2616, 2015

Tableau 3: Performances de croissance des poulets de chairs nourris aux résidus de semoule de manioc supplémentés par la spiruline.

\begin{tabular}{|c|c|c|c|c|c|c|c|c|c|c|c|c|c|}
\hline $\begin{array}{l}\text { Périodes } \\
\text { (jours) }\end{array}$ & $\mathbf{R 0}_{(0)}$ & $\mathbf{R 0}_{(0,5)}$ & $\mathbf{R 0}_{(\mathbf{1})}$ & $\mathbf{R 5 0}_{(0)}$ & R50 $_{(0,5)}$ & $\mathbf{R 5 0}_{(\mathbf{1})}$ & $\mathbf{R 7 5}_{(0)}$ & $\mathbf{R 7 5}_{(0,5)}$ & $\mathbf{R 7 5}_{(\mathbf{1})}$ & $\mathbf{R 1 0 0}_{(0)}$ & $\mathbf{R 1 0 0}_{(0,5)}$ & $\mathbf{R 1 0 0}_{(\mathbf{1})}$ & $\mathbf{P}$ \\
\hline \multicolumn{14}{|c|}{ Consommation alimentaire (g) } \\
\hline 01 à 21 & $\begin{array}{c}1271,62 \pm \\
156,94^{\mathrm{a}}\end{array}$ & $\begin{array}{c}1344,75 \pm \\
102,36^{\mathrm{a}}\end{array}$ & $\begin{array}{c}1443,75 \pm \\
132,03^{\mathrm{a}}\end{array}$ & $\begin{array}{c}1417,60 \pm \\
172,34^{\mathrm{a}}\end{array}$ & $\begin{array}{c}1492,78 \pm \\
193,74^{\mathrm{a}}\end{array}$ & $\begin{array}{c}1397,25 \pm \\
188,09^{\mathrm{a}}\end{array}$ & $\begin{array}{c}1422,30 \pm \\
90,51^{\mathrm{a}}\end{array}$ & $\begin{array}{r}1323,08 \pm \\
229,14^{\mathrm{a}}\end{array}$ & $\begin{array}{c}1376,23 \pm \\
255,31^{\mathrm{a}}\end{array}$ & $\begin{array}{c}1405,71 \pm \\
9,23^{\mathrm{a}}\end{array}$ & $\begin{array}{c}1320,87 \pm \\
116,56^{\mathrm{a}}\end{array}$ & $\begin{array}{c}1334,68 \pm \\
122,50^{\mathrm{a}}\end{array}$ & 0,811 \\
\hline 22 à 42 & $\begin{array}{c}3157,35 \pm \\
23,71^{\mathrm{b}}\end{array}$ & $\begin{array}{c}3445,49 \pm \\
212,77^{\mathrm{b}}\end{array}$ & $\begin{array}{c}3185,78 \pm \\
125,93^{\mathrm{ab}}\end{array}$ & $\begin{array}{c}2899,07 \pm \\
86,44^{\mathrm{b}}\end{array}$ & $\begin{array}{c}3018,48 \pm \\
17,91^{\mathrm{b}}\end{array}$ & $\begin{array}{c}3028,16 \pm \\
123,40^{\mathrm{b}}\end{array}$ & $\begin{array}{c}3024,43 \pm \\
39,52^{\mathrm{b}}\end{array}$ & $\begin{array}{c}2892,02 \pm \\
96,24^{\mathrm{b}}\end{array}$ & $\begin{array}{c}2920,59 \pm \\
36,00^{\mathrm{b}}\end{array}$ & $\begin{array}{c}2988,29 \pm \\
213,00^{\mathrm{b}}\end{array}$ & $\begin{array}{c}2889,38 \pm \\
81,59^{\mathrm{b}}\end{array}$ & $\begin{array}{c}2123,25 \pm \\
533,00^{\mathrm{c}}\end{array}$ & 0,000 \\
\hline \multirow[t]{2}{*}{01 à 42} & $\begin{array}{l}4428,97 \pm \\
143,09^{\text {abc }}\end{array}$ & $\begin{array}{c}4790,24 \pm \\
171,61^{\mathrm{a}}\end{array}$ & $\begin{array}{c}4629,53 \pm \\
193,91^{\mathrm{ab}}\end{array}$ & $\begin{array}{c}4316,67 \pm \\
235,49^{\text {bc }}\end{array}$ & $\begin{array}{l}4511,25 \pm \\
207,04^{\text {abc }}\end{array}$ & $\begin{array}{l}4425,41 \pm \\
222,10^{\text {abc }}\end{array}$ & $\begin{array}{c}4446,73 \pm \\
124,91^{\text {abc }}\end{array}$ & $\begin{array}{c}4216,00 \pm \\
319,91^{\mathrm{c}}\end{array}$ & $\begin{array}{c}4296,82 \pm \\
276,84^{\mathrm{bc}}\end{array}$ & $\begin{array}{c}4394,00 \pm \\
214,39^{\mathrm{bc}}\end{array}$ & $\begin{array}{c}4210,25 \pm \\
39,08^{\mathrm{c}}\end{array}$ & $\begin{array}{c}3457,93 \pm \\
446,96^{\mathrm{d}}\end{array}$ & 0,000 \\
\hline & \multicolumn{13}{|c|}{ Poids vif (g) } \\
\hline 01 à 21 & $\begin{array}{l}728,81 \pm \\
62,44^{\text {bc }}\end{array}$ & $\begin{array}{c}765,07 \pm \\
23,00^{\mathrm{ab}}\end{array}$ & $\begin{array}{c}812,62 \pm \\
24,52^{\mathrm{a}}\end{array}$ & $\begin{array}{l}699,07 \pm \\
55,03^{\mathrm{bc}}\end{array}$ & $\begin{array}{l}721,05 \pm \\
37,70^{\mathrm{bc}}\end{array}$ & $\begin{array}{c}665,33 \pm \\
44,65^{c}\end{array}$ & $\begin{array}{l}705,14 \pm \\
32,86^{\text {bc }}\end{array}$ & $\begin{array}{l}690,61 \pm \\
112,16^{\mathrm{bc}}\end{array}$ & $\begin{array}{c}661,95 \pm \\
76,64^{c}\end{array}$ & $\begin{array}{c}707,89 \pm \\
39,26^{\text {bc }}\end{array}$ & $\begin{array}{c}684,51 \pm 27 \\
02^{\mathrm{bc}}\end{array}$ & $690,55 \pm 19,95^{\mathrm{bc}}$ & 0,015 \\
\hline 01 à 42 & $\begin{array}{c}2108,42 \pm \\
12,28^{\mathrm{b}}\end{array}$ & $\begin{array}{c}2266,21 \pm \\
47,46^{\mathrm{a}}\end{array}$ & $\begin{array}{c}2234,09 \pm \\
71,80^{\mathrm{a}}\end{array}$ & $\begin{array}{c}1850,61 \\
\pm 115,35^{\text {ef }}\end{array}$ & $\begin{array}{c}2002,4 \pm \\
46,45^{\mathrm{c}}\end{array}$ & $\begin{array}{c}1952,50 \pm \\
66,95^{\text {cd }}\end{array}$ & $\begin{array}{c}1909,83 \pm \\
73,29^{\text {de }}\end{array}$ & $\begin{array}{c}1970,61 \pm \\
63,15^{\text {cd }}\end{array}$ & $\begin{array}{c}1831,22 \pm \\
9,54^{\mathrm{ef}}\end{array}$ & $\begin{array}{c}1774,34 \pm 8 \\
87^{\mathrm{f}}\end{array}$ & $\begin{array}{c}1861,86 \pm 36 \\
36^{\mathrm{ef}}\end{array}$ & $\begin{array}{c}1809,63 \pm \\
55,66^{\mathrm{f}}\end{array}$ & 0,000 \\
\hline \multicolumn{14}{|c|}{ Gain de poids (g) } \\
\hline 01 à 21 & $\begin{array}{c}686,01 \pm \\
62,44^{\mathrm{bc}}\end{array}$ & $\begin{array}{c}722,27 \pm \\
23,00^{\mathrm{ab}}\end{array}$ & $\begin{array}{c}769,82 \pm \\
24,52^{\mathrm{a}}\end{array}$ & $\begin{array}{c}656,27 \pm \\
55,03^{\text {bc }}\end{array}$ & $\begin{array}{c}678,24 \pm \\
37,70^{\text {bc }}\end{array}$ & $\begin{array}{c}622,53 \pm 44 \\
65^{\mathrm{c}}\end{array}$ & $\begin{array}{c}662,34 \pm \\
32,86^{\text {bc }}\end{array}$ & $\begin{array}{l}647,81 \pm \\
112,16^{\mathrm{bc}}\end{array}$ & $\begin{array}{c}619,15 \pm \\
73,64^{c}\end{array}$ & $\begin{array}{c}665,09 \pm \\
39,26^{\mathrm{bc}}\end{array}$ & $\begin{array}{c}641,71 \pm \\
27,02^{\text {bc }}\end{array}$ & $647,75 \pm 19,95^{\mathrm{bc}}$ & 0,015 \\
\hline 22 à 42 & $\begin{array}{c}1379,61 \pm \\
72,56^{\mathrm{bc}}\end{array}$ & $\begin{array}{c}1501,14 \pm \\
70,45^{\mathrm{a}}\end{array}$ & $\begin{array}{c}1421,46 \pm \\
95,94^{\text {ab }}\end{array}$ & $\begin{array}{c}1151,54 \pm \\
66,00^{\text {ef }}\end{array}$ & $\begin{array}{c}1281,37 \pm \\
47,96^{\mathrm{cd}}\end{array}$ & $\begin{array}{c}1287,17 \pm \\
52,29^{\text {cd }}\end{array}$ & $\begin{array}{c}1204,69 \pm \\
105,89^{\mathrm{de}}\end{array}$ & $\begin{array}{c}1280,00 \pm \\
49,17^{\text {cd }}\end{array}$ & $\begin{array}{c}1169,27 \pm \\
77,17^{\text {ef }}\end{array}$ & $\begin{array}{c}1066,45 \pm 45 \\
67^{\mathrm{f}}\end{array}$ & $\begin{array}{c}1177,35 \pm \\
60,87^{\mathrm{de}}\end{array}$ & $\begin{array}{c}1119,08 \pm \\
60,10^{\text {ef }}\end{array}$ & 0,000 \\
\hline 01 à 42 & $\begin{array}{c}2065,62 \pm \\
12,28^{\mathrm{b}}\end{array}$ & $\begin{array}{c}2223,41 \pm \\
4746^{\mathrm{a}}\end{array}$ & $\begin{array}{c}2191,25 \pm 71 \\
80^{\mathrm{a}}\end{array}$ & $\begin{array}{c}1807,81 \pm \\
115,35^{\text {ef }}\end{array}$ & $\begin{array}{c}1959,62 \pm \\
46,45^{\circ}\end{array}$ & $\begin{array}{c}1909,70 \pm \\
66,95^{\text {cd }}\end{array}$ & $\begin{array}{c}1867,03 \pm \\
73,29^{\text {de }}\end{array}$ & $\begin{array}{c}1927,81 \pm \\
63,15^{\text {cd }}\end{array}$ & $\begin{array}{c}1788,42 \pm \\
9,54^{2 f}\end{array}$ & $\begin{array}{c}1731,54 \pm \\
8,87^{\mathrm{f}}\end{array}$ & $\begin{array}{c}1819,06 \pm \\
36,36^{\mathrm{ef}}\end{array}$ & $1766,83 \pm 55,65^{\mathrm{f}}$ & 0,000 \\
\hline 01 à 21 & $1,85 \pm 0,12^{\mathrm{c}}$ & $1,86 \pm 0,13^{\mathrm{c}}$ & $1,88 \pm 0,20^{\mathrm{bc}}$ & $2,16 \pm 0,16^{\mathrm{abc}}$ & $2,22 \pm 0,40^{\mathrm{ab}}$ & \begin{tabular}{|c|} 
Indice de co \\
$2,25 \pm 0,28^{\mathrm{a}}$
\end{tabular} & $\frac{\text { ommation }}{2,16 \pm 0,25^{\text {abc }}}$ & $2,04 \pm 0,00^{\mathrm{abc}}$ & $2,22 \pm 0,30^{\mathrm{ab}}$ & $2,1 \pm 0,25^{\mathrm{abc}}$ & $\begin{array}{c}2,06 \pm \\
0,10^{\mathrm{abc}}\end{array}$ & $2,06 \pm 0,11^{\mathrm{abc}}$ & 0,081 \\
\hline 22 à 42 & $2,29 \pm 0,11^{\mathrm{b}}$ & $2,29 \pm 0,04^{\mathrm{b}}$ & $2,24 \pm 0,06^{\mathrm{b}}$ & $2,52 \pm 0,08^{\mathrm{b}}$ & $2,36 \pm 0,08^{\mathrm{b}}$ & $2,35 \pm 0,11^{\mathrm{b}}$ & $2,52 \pm 0,18^{\mathrm{b}}$ & $2,26 \pm 0,16^{\mathrm{b}}$ & $2,51 \pm 0,16^{\mathrm{b}}$ & $2,81 \pm 0,31^{\mathrm{a}}$ & $2,46 \pm 0,07^{\mathrm{b}}$ & $1,88 \pm 0,40^{\mathrm{c}}$ & 0,000 \\
\hline 01 à 42 & $2,14 \pm 0,07^{\mathrm{de}}$ & $2,15 \pm 0,04^{\text {cde }}$ & $2,11 \pm 0,06^{\mathrm{e}}$ & $2,39 \pm 0,09^{\mathrm{ab}}$ & $2,30 \pm 0,10^{\text {bcd }}$ & $2,32 \pm 0,10^{\mathrm{bc}}$ & $2,3 \pm 0,03^{\mathrm{ab}}$ & $2,18 \pm 0,10^{\text {cde }}$ & $2,40 \pm 0,15^{\mathrm{ab}}$ & $2,54 \pm 0,14^{\mathrm{a}}$ & $2,32 \pm 0,06^{\mathrm{bc}}$ & $1,95 \pm 0,21^{\mathrm{f}}$ & 0,000 \\
\hline & $\begin{array}{l}\text { c, d, e, f: les } \\
\text { bule de manio } \\
+50 \% \text { des ré } \\
\text { de spiruline; } \\
\text { oule de manio } \\
\text { robabilités }\end{array}$ & $\begin{array}{l}\text { nnes portan } \\
75(0)=25 \% \\
\text { de semoule } \\
=\text { ration té } \\
\% \text { de spirul }\end{array}$ & $\begin{array}{l}\text { mêmes lett } \\
+75 \% \text { des } \\
\text { anioc }+0 \text {, } \\
\text { avec } 100 \\
\mathrm{R}_{100_{(1)}}=10\end{array}$ & $\begin{array}{l}\text { la même } \\
\text { us de semo } \\
\text { espiruline; } \\
\text { naïs }+1 \% \\
\text { es résidus d }\end{array}$ & $\begin{array}{l}\text { ne sont pas s } \\
\text { e manioc; } \mathrm{R} \\
0,5)=25 \% \mathrm{~m} \\
\text { piruline, R50 }\end{array}$ & $\begin{array}{l}\text { cativement } \\
=100 \% \text { de } \\
75 \% \text { des ré } \\
=50 \% \text { maïs } \\
1 \% \text { de spi }\end{array}$ & $\begin{array}{l}\text { rentes }(\mathrm{P}> \\
\text { dus de sen } \\
\text { de semoul } \\
\% \text { des résid }\end{array}$ & $\begin{array}{l}\mathrm{R}_{0(0)}=\mathrm{Rati} \\
\text { le manioc } \\
\text { anioc }+0 \text {, } \\
\text { semoule de }\end{array}$ & $\begin{array}{l}\text { noin avec } \\
.5=\text { Ration } \\
\text { spiruline; } \\
\text { oc }+1 \% \mathrm{c}\end{array}$ & $\begin{array}{l}\text { de mais; } \mathrm{R} \\
100 \% \text { mai } \\
(0,5)=100 \% \\
\text { uline ; R75 }\end{array}$ & $\begin{array}{l}=50 \% \text { maïs }+ \\
, 5 \% \text { de spiru } \\
\text { résidus de se } \\
25 \% \text { maïs }+\end{array}$ & $\begin{array}{l}\% \text { des résidus de } \\
\text { e; } \mathrm{R} 50_{(0,5)}=50 \% \\
\text { oule de manioc }+ \\
\% \text { des résidus de }\end{array}$ & \\
\hline
\end{tabular}


J. R. KANA et al. / Int. J. Biol. Chem. Sci. 9(5): 2607-2616, 2015

Tableau 4: Rendement carcasse et poids des organes (\%) des poulets de chair nourris par des taux croissants des résidus de semoule de manioc supplémentés par la spiruline.

\begin{tabular}{|c|c|c|c|c|c|c|c|c|c|c|c|c|c|}
\hline \multicolumn{14}{|c|}{ Traitements } \\
\hline Paramètres & $\mathbf{R} \mathbf{0}_{(\mathbf{0})}$ & $\mathbf{R 0 _ { ( 0 , 5 ) }}$ & $\mathbf{R 0 _ { ( 1 ) }}$ & $\mathbf{R 5 0}_{(0)}$ & $\mathbf{R 5 0}_{(0,5)}$ & $\mathbf{R 5 0}_{(\mathbf{1})}$ & $\mathbf{R 7 5}_{(0)}$ & $\mathbf{R 7 5}_{(0,5)}$ & $\mathbf{R 7 5}_{(\mathbf{1})}$ & $\mathbf{R 1 0 0}_{(0)}$ & $\operatorname{R100}_{(0,5)}$ & $\mathbf{R 1 0 0}_{(1)}$ & $\mathbf{P}$ \\
\hline \multirow{2}{*}{$\begin{array}{l}\text { Rendement } \\
\text { carcasse }\end{array}$} & $73,27 \pm$ & $73,43 \pm$ & $72,86 \pm$ & $72,89 \pm$ & $73,51 \pm$ & $72,33 \pm$ & $71,56 \pm$ & $71,99 \pm$ & $71,48 \pm$ & $71,23 \pm$ & $71,94 \pm$ & $70,95 \pm$ & 0,108 \\
\hline & $1,13^{\mathrm{ab}}$ & $2,15^{\mathrm{a}}$ & $2,03^{\mathrm{ab}}$ & $1,09^{\mathrm{ab}}$ & $1,35^{\mathrm{a}}$ & $1,58^{\mathrm{ab}}$ & $2,54^{\mathrm{ab}}$ & $1,07^{\mathrm{ab}}$ & $2,05^{\mathrm{ab}}$ & $4,21^{\mathrm{ab}}$ & $0,80^{\mathrm{ab}}$ & $1,21^{\mathrm{b}}$ & \\
\hline \multirow[t]{2}{*}{ Pattes } & $3,52 \pm$ & $4,79 \pm$ & $3,71 \pm$ & $4,08 \pm$ & $3,84 \pm$ & $3,87 \pm$ & $4,07 \pm$ & $3,64 \pm$ & $3,93 \pm$ & $4,05 \pm$ & $3,91 \pm$ & $3,99 \pm$ & 0,540 \\
\hline & $0,32^{b}$ & $14,66^{\mathrm{a}}$ & $0,46^{\mathrm{b}}$ & $0,36^{\mathrm{ab}}$ & $0,43^{\mathrm{ab}}$ & $0,55^{\mathrm{ab}}$ & $0,38^{\mathrm{ab}}$ & $0,37^{\mathrm{b}}$ & $0,48^{\mathrm{ab}}$ & $0,63^{\mathrm{ab}}$ & $0,34^{\mathrm{ab}}$ & $0,40^{\mathrm{ab}}$ & \\
\hline \multirow[t]{2}{*}{ Foie } & $2,27 \pm$ & $2,05 \pm$ & $1,9 \pm$ & $2,17 \pm$ & $1,94 \pm$ & $2,12 \pm$ & $2,37 \pm$ & $2,14 \pm$ & $2,12 \pm$ & $2,15 \pm$ & $2,36 \pm$ & $2,23 \pm$ & 0,303 \\
\hline & $0,19^{\mathrm{ab}}$ & $0,30^{\mathrm{ab}}$ & $0,31^{\mathrm{ab}}$ & $0,36^{\mathrm{ab}}$ & $0,31^{\mathrm{b}}$ & $0,19^{\mathrm{ab}}$ & $0,56^{\mathrm{a}}$ & $0,44^{\mathrm{ab}}$ & $0,38^{\mathrm{ab}}$ & $0,39^{\mathrm{ab}}$ & $0,28^{\mathrm{a}}$ & $0,26^{\mathrm{ab}}$ & \\
\hline \multirow[t]{2}{*}{ Cour } & $0,57 \pm$ & $0,46 \pm$ & $0,56 \pm$ & $0,75 \pm$ & $0,52 \pm$ & $0,57 \pm$ & $0,57 \pm$ & $0,55 \pm$ & $0,53 \pm$ & $0,49 \pm$ & $0,63 \pm$ & $0,57 \pm$ & 0,000 \\
\hline & $0,15^{\mathrm{bc}}$ & $0,11^{\mathrm{c}}$ & $0,09^{\mathrm{bc}}$ & $0,08^{\mathrm{a}}$ & $0,09^{\mathrm{bc}}$ & $0,08^{\mathrm{bc}}$ & $0,11^{\mathrm{bc}}$ & $0,11^{b c}$ & $0,03^{\mathrm{bc}}$ & $0,11^{\mathrm{c}}$ & $0,13^{\mathrm{b}}$ & $0,11^{\mathrm{bc}}$ & \\
\hline \multirow[t]{2}{*}{ Pancréas } & $0,23 \pm$ & $0,23 \pm$ & $0,26 \pm$ & $0,39 \pm$ & $0,24 \pm$ & $0,31 \pm$ & $0,19 \pm$ & $0,25 \pm$ & $0,24 \pm$ & $0,20 \pm$ & $0,21 \pm$ & $0,22 \pm$ & 0,015 \\
\hline & $0,08^{\mathrm{b}}$ & $0,07^{\mathrm{b}}$ & $0,11^{\mathrm{b}}$ & $0,18^{\mathrm{a}}$ & $0,13^{\mathrm{b}}$ & $0,08^{\mathrm{ab}}$ & $0,06^{\mathrm{b}}$ & $0,11^{\mathrm{b}}$ & $0,09^{b}$ & $0,05^{\mathrm{b}}$ & $0,11^{\mathrm{b}}$ & $0,07^{\mathrm{b}}$ & \\
\hline
\end{tabular}

$\mathrm{a}, \mathrm{b}, \mathrm{c}$ : les moyennes portant les mêmes lettres sur la même ligne ne sont pas significativement différentes $(\mathrm{P}>0,05)$. $\mathrm{R}_{0(0)}=$ Ration témoin avec $100 \%$ de mais; $\mathrm{R}_{50(0)}=50 \%$ maïs $+50 \%$ des résidus $\mathrm{d}$ 作 semoule de manioc $; \mathrm{R}_{75(0)}=25 \%$ maïs $+75 \%$ des résidus de semoule de manioc; $\mathrm{R}_{100(0)}=100 \%$ des résidus de semoule de manioc ; $\mathrm{R} 0_{(0,5)}=\mathrm{Ration}$ témoin $100 \%$ mais $+0,5 \%$ de spiruline; $\mathrm{R} 50_{(0,5)}=50 \%$ maïs $+50 \%$ des résidus de semoule de manioc $+0,5 \%$ de spiruline; $R 75_{(0,5)}=25 \%$ maïs $+75 \%$ des résidus de semoule de manioc $+0,5 \%$ de spiruline $; \mathrm{R} 100_{(0,5)}=100 \%$ des résidus de semoule de manioc + $0,5 \%$ de spiruline; $\mathrm{R} 0_{(1)}=$ ration témoin avec $100 \%$ de maïs $+1 \%$ de spiruline, $\mathrm{R} 50(1)=50 \%$ maïs $+50 \%$ des résidus de semoule de manioc $+1 \%$ de spiruline $; \mathrm{R} 75(1)=25 \%$ maïs $+75 \%$ des résidus de semoule de manioc $+1 \%$ de spiruline; $\mathrm{R} 100_{(1)}=100 \%$ des résidus de semoule de manioc $+1 \%$ de spiruline $; \mathrm{P}=$ Probabilités. 


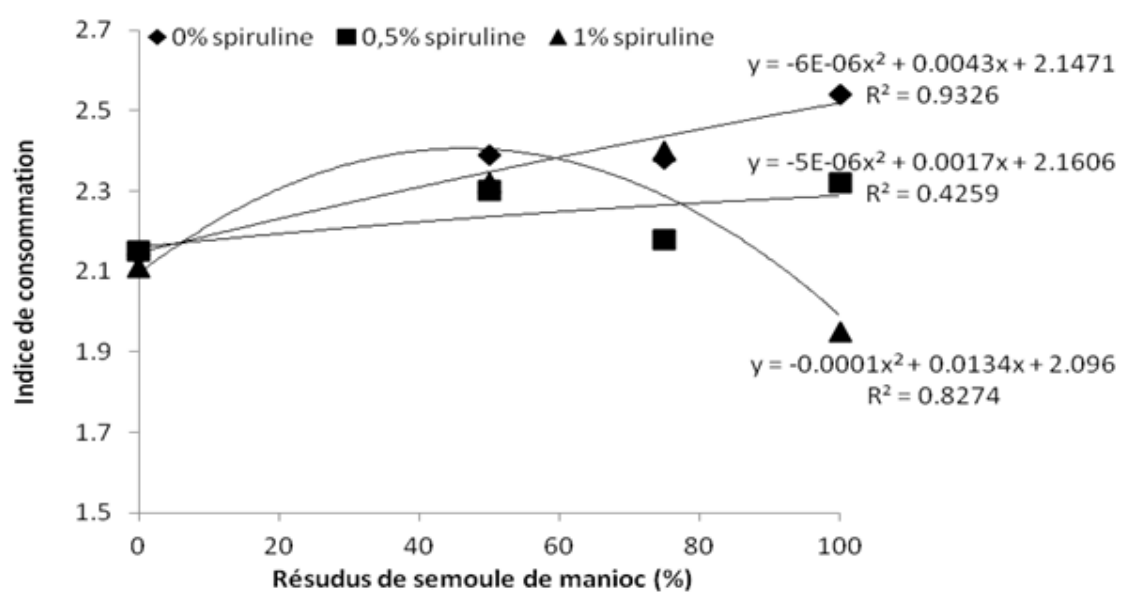

Figure 1: Régression de l'indice de consommation sur le taux d'incorporation des résidus de semoule de manioc supplémentés par la spiruline.

\section{DISCUSSION}

L'incorporation de la spiruline dans l'aliment témoin a induit une augmentation non significative $(\mathrm{P}>0,05)$ de la consommation alimentaire cumulée comparée à la ration sans spiruline. Un rapport similaire a été dressé par Bujard et al. (1970) selon lequel l'incorporation de 0,5 à $1,5 \%$ de spiruline dans la ration des volailles améliorait la qualité organoleptique de l'aliment et entraînait une augmentation non significative de la consommation. La tendance à consommer plus d'aliment contenant la spiruline pourrait être attribuée à la bonne digestibilité des nutriments contenus dans la spiruline et à l'absence des parois cellulosique chez cette algue qui facilite sa digestibilité. En effet, d'après Costa et al. (2002) la faible teneur en cellulose explique la forte digestibilité (75 à 83\%) de la spiruline qui ne nécessite pas une cuisson ni un traitement spécial pour une bonne digestibilité protéique. L'incorporation de la spiruline à des taux de 0,5 et $1 \%$ dans la ration contenant uniquement du maïs (R0) et la ration contenant $50 \%$ de résidus de semoule R50 a permis d'enregistrer les performances pondérales plus élevées $(\mathrm{P}<0,5)$ que ces mêmes rations sans spiruline. Ces observations sont en contradiction avec celles de Razafindrajaona et al. (2008) qui avaient incorporé $50 \mathrm{mg} / \mathrm{kg}$ et $100 \mathrm{mg} / \mathrm{kg}$ de spiruline dans les provendes et n'ont enregistré aucun effet significatif sur la croissance pondérale des poulets de chair mais a permis d'optimiser la production quantitative et qualitative de viande de poulet. Ces mêmes auteurs ont rapporté que la consommation de la spiruline a entraîné un meilleur développement des différents quartiers de l'animal (carcasse) et ils ont conclu que l'incorporation de la spiruline dans les provendes permet d'améliorer non seulement sa qualité nutritionnelle mais aussi son efficacité biologique.

La tendance à la baisse du poids des poulets avec le taux d'incorporation croissant des résidus de semoule supplémentés ou non par la spiruline serait le reflet de la faible digestibilité de l'aliment liée à l'augmentation de sa viscosité. Ce résultat corrobore celui de Razafindrajaona et al. (2008) qui, après avoir supplémenté les rations des jeunes souris femelles avec $20 \mathrm{mg} / \mathrm{kg}$ et $500 \mathrm{mg} / \mathrm{kg}$ de poids vif avec la spiruline, ont enregistré un effet légèrement négatif et défavorable sur la croissance. Les travaux de Mafouo et al. (2011) portant sur la substitution partielle ou totale du maïs par la farine de manioc dans l'aliment des poulets de chair ont abouti à cette même conclusion. Cependant, les poids obtenus dans cette étude sont supérieurs à ceux obtenus par ces derniers auteurs. Cette 
différence serait liée à l'amélioration de la texture des résidus de semoule de manioc lors $\mathrm{du}$ processus industriel de production de la semoule et à l'incorporation de la spiruline dans l'aliment qui aurait amélioré la valeur nutritive et l'efficacité d'utilisation digestive de l'aliment. D'après Tayomizu et al. (2001), la richesse de la spiruline en acides aminés permet à la race à viande de produire une chair de qualité et en quantité optimale. Les résultats de la présente étude sont aussi contraires à ceux obtenus avec d'autres monogastriques tels que le porc. En effet, Fevrier et Seve (1976) n'ont enregistré aucun effet positif sur la croissance des porcelets précocement sevrés avec l'introduction de $12 \%$ de spiruline dans la ration. Selon les mêmes auteurs, l'adjonction de $5 \%$ de spiruline dans la ration de truies reproductrices n'affecte ni la prolificité ni la croissance ultérieure des animaux. Ils ont en fin conclut que la spiruline ne peut pas être considérée comme protéine de base des rations pour animaux. Par contre, Ramaroson (2003) a rapporté qu'avec une dose de 2,5 g par jour par tête dans la ration des porcelets mâles de race Large White sevrés à 7 semaines et castrés, la spiruline agit en tant que facteur de croissance. Selon ce dernier auteur, la richesse en acides aminés de la spiruline favorise la synthèse de n'importe quelles enzymes utiles au bon fonctionnement $\mathrm{du}$ corps de l'animal. Sa consommation le rend plus vigoureux, productif et totalement émancipé (Razafindrajaona et al., 2010), surtout lors de sa phase de croissance préliminaire. Cette conclusion rejoint celle de Brum et al. (1990) qui ont recommandé qu'à faible dose (inférieure à 5\%), la spiruline est une source protéique intéressante pour les oiseaux en croissance.

Lorsqu'on considère le même taux de résidus de semoule dans l'aliment, le rendement carcasse, le poids relatif de la tête, des pattes, du foie, du cœur, du pancréas et de la graisse abdominale évoluent en dents de scie entre les traitements. Ce résultat se rapproche de ceux rapportés par Mafouo et al. (2011) et Kana et al. (2014) qui ont respectivement rapporté des résultats similaires en substituant le maïs à $50 \%, 75 \%$ et $100 \%$ avec la farine de manioc et les résidus de semoule de manioc dans l'aliment des poulets de chair.

\section{Conclusion}

Bien que dépourvue de paroi cellulosique et contenant les principaux acides aminés, vitamines et minéraux dont l'animal à besoin en quantité considérable, la spiruline n'a pas facilité de façon significative l'utilisation des résidus de semoule de manioc par le poulet de chair. En effet, tel que relevé par d'autres études, en plus de sa pauvreté en nutriments essentiels, le manioc et ses sousproduits sont très peu digestes. Il serait souhaitable d'apporter un supplément de méthionine et de lysine synthétiques pour améliorer la valeur biologique de la protéine de la ration et les enzymes fibrinolytiques pour faciliter la digestion de ce sous-produit.

\section{CONFLIT D'INTÉRÊT}

Les auteurs ne déclarent aucun conflit d'intérêt.

\section{CONTRIBUTION DES AUTEURS}

JRK a supervisé la collecte, le traitement des données et a rédigé le premier manuscrit ; ADH, TRN et KHM ont collecté et traité les données; AT, YT et HRZ ont relu et corrigé le manuscrit.

\section{REFERENCES}

Awah-Ndukum J, Téguia A, Defang HF, Awungnjia A. 2008. The effect of replacing maize with dred cassava peels on growth performance of broiler chickens. Sci. Agro. Dévelop., 4(1): 4855.

Bujard EU, Braco-U, Mauron J, Mottu F, Nabholz A, Wuhrmann JJ, Clément. 1970. Composition and nutritive value of blue green algae (Spirulina) and their possible use in food formulations. $3^{\text {rd }}$ International Congress of Food Science and Technology, Washington. 
Brum JC, Leclerc B, Lessire M, Perez JM. 1990. L'alimentation des Animaux Monogastriques. INRA: Paris. 282p.

Costa JAV, Colla LM, Duarte P, Kabke K, Weber A. 2002. Modelling of Spirulina platensis growth in fresh water using response surface methodology. World J. Microb. Biotech., 18: 603-607. DOI 10.1023/A:1016822717583

Fevrier C, Sève B. 1976. Incorporation of Spirulina (Spirulina maxima) into pig diets. Ann. Nutr. Aliment., 29: 625 -6 30.

Jourdain. 1980. L'aviculture en Milieu Tropical. Jourdain International : Couloumiers ; 43-45.

Jourdan JP. 1996. Cultivez Votre Spiruline. Ed. Antenna Technologies : Genève ; 143.

Kana JR, Tadjong RN, Kuietche HM, Tefack Y, Zambou H, Teguia A. 2014: Valorisation des résidus de manioc en substitution du maïs dans la ration alimentaire du poulet de chair. Livestock Research for Rural Development. 26: 48. Retrieved June 3, 2015, from http://www.lrrd.org/lrrd26/3/kana26048.h tm

Mafouo NH, Teguia A, Kana JR, Mube HK, Diarra M. 2011. Effet du niveau d'incorporation de la farine de manioc dans la ration sur les performances de croissance des poulets de chair. Livestock Research for Rural Development. 23: 76. Retrieved April 23, 2011, from http://www.lrrd.org/lrrd23/4/mafo23076. htm
Pierlovisic. 2007. L'Homme et la Spiruline: Un avenir commun? Thèse de Doctorat en pharmacie, Université de Paris V, 32p

Ramaroson C. 2003. Influence de l'incorporation de doses faibles de spiruline dans l'alimentation de porcelet sevré à l'âge de deux mois. Mémoire de fin d'études, Département Elevage, ESSA Antananarivo, Madagascar, 30p.

Razafindrajaona JM, Rokotozandriny JN, Raphaël Rakotozandrindrainy, Antsivasoa Tsivingaina, Kotonirina Ramapiherika, Randria JN. 2008. Influence de l'incorporation dans les provendes de la Spiruline de Madagascar (Spirulina platensis var. toliara) sur la croissance des poulets de chair. Colloque International sur la Spiruline, Toliara, 28, 29 et 30 avril 2008.

Razafindrajaona JM, Rokotozandriny JN, José NR, Ramampiherika KD. 2010. Etude de la performance nutritionnelle de la Spiruline de Madagascar (Spirulina platensis variété Toliarensis) sur la souris. ERAILS Madagascar, 18/06/2010.

Toyomizu M, Sato K, Taroda H, Akiba Y. 2001. Effects of dietary Spirulina on meat colour in muscle of broiler chickens. Brit. Poult. Sci., 42: 197-202. DOI:10.1080/00071660120048447 